\title{
La protección social en una sociedad de semejantes ${ }^{*}$
}

\author{
Robert Castel
}

\begin{abstract}
The changes, that during the last three decades have affected the labor laws and the protections associated with them, have created a growing sensation of insecurity in western societies. Professor Castel, taking as example the French situation, examines this phenomenon and proposes, in the first place, a basic distinction between civil insecurity and social insecurity. Then the author focuses on the latter type of insecurity and reflects on the economic and social changes that are involved in the current preponderance of flexible jobs. This article, therefore, calls of attention to the risks involved in a unilateral and simplistic view of society and the State in addition to the very important role played by social property in the development of a true democracy.
\end{abstract}

Si he comprendido bien la solicitud que me han hecho, se trata de que juntos reflexionemos sobre la pregunta ¿qué quiere decir estar protegidos en una sociedad moderna? En qué condiciones es posible vencer la inseguridad, que ha sido y sigue siendo, en gran medida, un factor permanente de la vida en sociedad. Thomas Hobbes dio, a principios de la modernidad, es decir a finales del siglo XVII, una imagen bastante espantosa, pero al mismo tiempo realista, de lo que sería una sociedad sin leyes, sin protección y librada a la competencia de todos contra todos. Se podría decir que la historia de las civilizaciones ha sido, en gran medida, un esfuerzo por establecer reglas, protecciones, un orden social que impida que los hombres se enfrenten de manera 
permanente en conflictos. Es un esfuerzo frágil, que siempre ha estado amenazado, de manera que no se puede hablar de seguridad sin hablar de inseguridad, de las amenazas que pesan sobre la protección de los individuos. Sobre esta relación entre seguridad e inseguridad quiero centrar el propósito de esta conferencia, haciendo énfasis en la importancia fundamental de la protección social como un factor determinante que garantiza la cohesión de una sociedad y la solidaridad entre sus miembros. Y también haciendo énfasis en la fuerza de las dinámicas económicas que hoy van en contra de esta necesidad de protección.

Las dos intervenciones que me han solicitado — la de hoy y la de mañana — se pueden considerar como complementarias en la exposición de este propósito. Por esta razón, les propongo centrar ahora mi intervención sobre las dificultades que se presentan en una sociedad moderna para estar protegidos, por el número de factores de inseguridad que hoy tienden a multiplicarse. De manera particular, insistiré en lo que se puede llamar un aumento de la inseguridad social, que me parece una característica fundamental de la actual situación social, al menos en los países de Europa. Luego, en una segunda parte, es decir mañana, trataré de establecer las razones y las consecuencias de este aumento de la inseguridad social. Para decirlo de manera rápida, me parece que estas razones provienen de un nuevo régimen del capitalismo al que entramos hace unos veinte años y que, a diferencia del capitalismo industrial precedente, se caracteriza por una competencia exacerbada en el marco de una economía mundializada que cuestiona precisamente el sistema de protección que se había construido durante el capitalismo industrial. Las consecuencias son una degradación de la condición de los trabajadores y también una degradación del estatus de un cierto número de individuos. Este es, de manera muy esquemática, el marco general de mi presentación, a la que trataré de dar más consistencia en estas dos exposiciones.

\footnotetext{
* Este artículo es la versión traducida al español de dos conferencias que el profesor Robert Castel dictó en la Universidad Icesi de Cali, durante el congreso sobre Protección Social realizado en el mes de abril de 2006. Fue revisado, corregido y autorizado para su publicación en esta revista por el propio autor.
} 
Antes que nada debo hacer una observación. Desafortunadamente, no conozco muy bien la situación de Colombia como para poder partir de ella para argumentar mi propósito. Me veré obligado entonces a apoyarme en situaciones que conozco, es decir, en la situación francesa y un poco más en general sobre la situación europea. Pero soy muy consciente de las enormes diferencias con relación a lo que sucede aquí en Colombia. Tratándose de cuestiones sobre protección, de seguridad e inseguridad, nosotros en Europa somos muy privilegiados. Cuando hable de Francia o de Europa, de la inseguridad social, de la ausencia de protección, ustedes estarán en todo el derecho de decir que no tenemos razón para quejarnos, y que si en Colombia se diera el equivalente de esas mismas condiciones la gente estaría mucho más contenta. Pienso que es cierto. Pero voy a insistir sobre el hecho de que no presento la situación francesa como una especie de modelo, sino que es más bien una forma de organización de la protección social que ha sido construida a lo largo de una extensa historia que tiene una especificidad, tiene cualidades y defectos. El hecho de presentarla hoy puede darnos, al menos así espero, materia de reflexión sobre las dinámicas de la protección social, sobre las exigencias y las dificultades para establecer la seguridad, y sin dejar de tener en cuenta la diferencia con lo que sucede aquí. No contamos con el tiempo, y tampoco tendría los medios, para hacer un análisis comparativo entre la situación en Francia y la situación en Colombia, pero voy a tratar de dar algunas informaciones para que podamos discutir sobre el sentido de lo que voy a tratar de decir sobre la pregunta: ¿qué significa estar protegidos? Del sentido que puede tener en relación con lo que sucede aquí, incluso si es por oposición a la situación de Francia y no solamente con base en analogías y aproximaciones. En todo caso, espero que podamos dar juntos esta discusión.

Para poder hacer ver la dificultad de reflexionar sobre la pregunta ¿qué significa estar protegidos? voy a partir de una paradoja que, según creo, caracteriza la situación francesa y que muestra también la gran diferencia con la situación colombiana. Francia goza de una protección social amplia y también de una gran seguridad civil política. No obstante, el sentimiento de inseguridad se ha convertido en Francia, a lo largo de los últimos años, en una preocupación muy grande, por no decir en una obsesión. Por ejemplo, las campañas políticas se han polarizado mucho en torno a este tema de la inseguridad. A tal punto, que 
para las últimas elecciones presidenciales en Francia en el 2002, el candidato de extrema derecha, que representaba precisamente esa especie de obsesión por la seguridad, por así decir, se ubicó en las elecciones por encima del candidato de la izquierda que era el primer ministro socialista. Evoco este episodio de la vida política francesa para invitarlos a reflexionar un poco sobre la complejidad del asunto de la inseguridad y de las protecciones. El sentimiento de inseguridad, de sentirse en inseguridad, no equivale a una ausencia de protección. Esto se debe tal vez a que, aunque tenemos y disponemos aún de protección, tenemos la sensación de que las protecciones son frágiles, que están amenazadas y que tenemos miedo de perderlas. Creo que está muy generalizada hoy, al menos en Francia, esta sensación de un ascenso de la inseguridad social, sobre el cual volveré más adelante. Además, el hecho de sentirse desprotegido puede venir de que, habiendo sido controlados ciertos riesgos, nos volvemos sensibles a nuevos riesgos. Por ejemplo, para la humanidad el gran riesgo alimenticio era antes el hambre. Hoy todavía lo es en algunos sitios. El riesgo de no tener nada que comer. En todo caso, en Francia este riesgo ha sido controlado. Nadie en Francia se muere de hambre y eso es bueno. Pero podríamos decir que el miedo se ha desplazado y que ahora el miedo está en el plato. Es, por ejemplo, el miedo de tragarse el prion, la desdichada proteína transmisora de la enfermedad de las vacas locas, o la gripe aviaria, o uno de esos productos cancerígenos de los que se dice que la lista crece todos los días. Vemos pues que, para algunos, el miedo de comer ha reemplazado al miedo de no tener nada que comer. Escogí este ejemplo que es un poco exagerado tal vez, para ilustrar lo que considero un aspecto importante de la problemática de la seguridad. En el fondo no hay riesgos en sí o inseguridad en sí. Todo depende del contexto, de las condiciones sociales o históricas en las que uno pueda sentirse protegido o no. Esta sensación de inseguridad también puede resultar del hecho de que uno amalgama distintos tipos de riesgos: el miedo de ser agredido en la calle o de ser tomado como rehén por un comando, el miedo de ser víctima del terrorismo, por ejemplo, pero también el miedo de que la situación social se degrade, de perder el empleo; también se puede tener miedo de las catástrofes de tipo ecológico, del recalentamiento del planeta, de los riesgos sanitarios, etc. Se comprende entonces que algunos hayan podido decir que vivimos en una sociedad del 
riesgo. Retomo el título de un libro del sociólogo alemán Ulrich Beck, quien tuvo mucho éxito con su obra La sociedad del riesgo. Esto quiere decir que, en el fondo, el futuro se leería ensombrecido por el signo de la amenaza. Ulrich Beck llega a decir que vivimos en este planeta como sobre un asiento eyector.

Esto es un retroceso extraordinario con relación a lo que prevalecía hace unos treinta años, al menos en Europa, con la creencia de que el futuro sería mejor que el presente. Es lo que, por otra parte, se conoce como progreso social o, para muchos, el mañana concebido bajo el signo de la revolución, de un mañana feliz. Hoy el número de personas que piensan que el mañana será mejor es cada vez menor.

No comparto las convicciones un poco catastróficas sobre el futuro, como las de Ulrich Beck. Pienso que este tipo de discurso mantiene el miedo y conduce a la impotencia y a la resignación. Creo que es necesario desinflar este sentimiento generalizado de inseguridad. Para eso, creo que hay que descomponerlo; es necesario distinguir los diferentes factores de inseguridad, cuya amalgama puede conducir a la confusión y a la impotencia para enfrentar la inseguridad. Desde este punto de vista, me parece importante diferenciar dos tipos de inseguridad: la inseguridad civil y la inseguridad social. Es algo simple, pero pienso que es importante.

La inseguridad civil agrupa, a grandes rasgos, los problemas que crea la delincuencia. El ejercicio de la violencia física, lo que atenta contra la integridad de las personas, de sus bienes; es decir, las agresiones físicas, los crímenes, los golpes y las heridas, las violaciones, las degradaciones, y podríamos agregar los raptos, los secuestros, la toma de rehenes, etc. En las sociedades modernas, la lucha contra esta violencia, que atenta contra los bienes y las personas, hacía parte y hace parte, de una función esencial del Estado. Es lo que se denomina el Estado de Derecho, que en principio tiene el monopolio de la lucha contra esta inseguridad civil. Este Estado se ha dotado de instituciones especializadas para hacerlo, en particular, la policía y la justicia. En el fondo, esta conservación de la seguridad civil es en principio la base de un Estado de Derecho que se sigue, por ejemplo, de la Declaración de los Derechos del Hombre y del Ciudadano, que pone en el nivel de los derechos inalienables sagrados la integridad de la persona, del individuo y de sus bienes. 
Creo que hay una toma de conciencia de que la vida en sociedad pasa por el respeto de la persona y de sus bienes y que no se puede realmente construir una sociedad, en el sentido amplio de la palabra, con los semejantes si se vive bajo la amenaza permanente de la agresión, de la violencia física, del robo o de la violación, y con mayor razón, si se vive en un contexto casi de guerra civil. Ustedes tienen, sin duda, muchas cosas que decir sobre este punto, porque Colombia aparece como un país que ha tenido muchas dificultades para mantener la paz civil e imponer condiciones de Estado de Derecho. Les recuerdo esto para decirles también que soy consciente de la situación privilegiada de Francia y de los principales países de Europa Occidental con relación a esta situación.

Pero también en un país como Francia quedan problemas y en los últimos años hemos podido observar un aumento de lo que denomino la inseguridad civil como, por ejemplo, un incremento del índice de delincuencia, de violencia contra las personas y, en particular, en el caso de los suburbios, los barrios periféricos de las grandes ciudades. Ustedes saben, seguramente, que hace poco hubo carros incendiados, peleas entre bandas de jóvenes y la policía, etc. Eso genera mucho miedo en Francia. La sensación de que habría en la sociedad francesa unas zonas de no-derecho, barrios que estarían dedicados a la violencia urbana. Esto plantea un terrible interrogante con relación al principio del Estado de Derecho, porque dada esta preocupación, se exige un aumento de la autoridad del Estado. Pero este aumento de autoridad que se exige al Estado ¿se puede ejercer dentro del marco del respeto de la ley, en el Estado de Derecho? Se podría decir lo mismo, sin lugar a dudas, de los Estados Unidos y su forma de realizar lo que ellos denominan la guerra contra el terrorismo. Esto plantea un interrogante (dilema, paradoja): países como Francia o los Estados Unidos proclaman su adhesión a los derechos del hombre hasta el punto de dar, al respecto, con frecuencia, lecciones al resto del mundo, pero al mismo tiempo se encuentran en riesgo de caer en situaciones de no-derecho o en todo caso de restricciones de las libertades públicas. Hay un problema real, porque, es legítimo luchar contra la delincuencia, contra la violencia en todas sus formas, porque la paz civil es una condición esencial para vivir en una sociedad democrática; pero si una democracia es un Estado de Derecho, en el cual el poder del Estado está limitado por la ley, puede resultar difícil que 
sea eficaz en esta lucha. Creo que es, en todo caso, un problema que se presenta también en Francia, en donde muchas personas tienen la sensación de no estar suficientemente protegidas contra este tipo de violencia. Lo que mantiene precisamente esta sensación generalizada de inseguridad y que a ustedes les parece exagerado, a mí también me parece exagerado, pero es una sensación que existe. Esta sensación relacionada con la inseguridad civil no se debe confundir con ese otro tipo de inseguridad que es la inseguridad social. Creo que no hay que confundirlas, aunque las dos pueden adicionarse.

Entonces, ¿en qué consiste está inseguridad social? Yo diría que sentirse en inseguridad social es estar a merced del más mínimo azar en la existencia, y que una enfermedad, un accidente, una interrupción del trabajo puedan hacernos trastabillar. Se encuentra uno sin recursos, enfrentado a la angustia de no saber qué le depara el futuro. Hay una vieja expresión que se refiere a esto: "vivir el día a día”. En este sentido, la inseguridad social ha sido un factor permanente y se encuentra todavía presente, en muchos países, en gente cuya condición cae bajo la denominación de “el pueblo”. Es decir, las personas que, por ejemplo, sólo tenían su trabajo para vivir o seguir adelante y que estaban a merced del mínimo accidente y perdían la capacidad para satisfacer sus deseos y los de su familia y además, corrían el riesgo de caer en la degradación social. Creo que eso era y sigue siendo la inseguridad social. Podríamos hablar mucho sobre esto porque desafortunadamente esta situación existe en alto grado. Pero en todo caso, en algunos países, entre los que está Francia, esta inseguridad social había sido controlada, en lo esencial, precisamente, mediante el establecimiento de sistemas amplios de protección: las protecciones sociales, la seguridad social, en el sentido amplio del término.

La lucha contra esta inseguridad social ha estado relacionada con otra función del Estado, diferente del Estado de Derecho que mencioné anteriormente, y es lo que llamamos el Estado Providencia o Estado Social que se implantó tardíamente en Europa Occidental, a partir de finales del siglo XIX y sobre todo en el período posterior a la Segunda Guerra Mundial. Podríamos decir que la función esencial de este Estado Social había sido la de actuar como una especie de reductor de los riesgos sociales. Fue así como se logró vencer, en lo esencial, la inseguridad social. Los seguros contra accidentes, contra la enfermedad, 
contra las interrupciones del trabajo, contra este riesgo dramático que se presentaba para el trabajador que ya era demasiado viejo para trabajar, el riesgo de encontrarse completamente desprovisto de recursos. Para tomar solamente este ejemplo, creo que podemos decir que el derecho a la jubilación ha sido un medio extraordinario de lucha contra la inseguridad social, puesto que, antes de este derecho, la situación del trabajador viejo que no podía seguir trabajando era con frecuencia espantosa. Con el derecho a la jubilación el trabajador no se convirtió en un propietario rico, pero con eso dispone de derechos a unos recursos mínimos que le garantizan cierta independencia social. Entonces, salió de la inseguridad social gracias a este derecho.

Se podría generalizar diciendo que a partir de este derecho a la jubilación se desarrolló todo un conjunto de protecciones relacionadas con la condición del trabajador, y este desarrollo de sistemas de protección fue tal que en Europa se pudo hablar, en los años 60 y 70, de sociedades aseguradoras. Es decir, sociedades en las que la gran mayoría de la población estaba protegida contra los principales riesgos sociales. En resumen, sociedades en las que la seguridad social había reemplazado a la inseguridad social para la mayoría de los ciudadanos, con la garantía del Estado. Por esta razón se podía hablar de victoria sobre la inseguridad social en este tipo de formaciones sociales. Victoria que, sin duda, no era completa puesto que todavía quedaban algunos individuos y grupos de individuos un poco marginales que no estaban incluidos en este sistema de protección. En Europa, en los años 60 y 70, se pensaba en general que la pobreza que quedaba en las sociedades era una pobreza residual que se absorbería gracias precisamente a esta búsqueda del progreso social. Desafortunadamente no fue lo que sucedió y a partir de 1970, más o menos, se presenta en Europa un bloqueo de esta dinámica que había caracterizado sobre todo la época posterior a la Segunda Guerra Mundial, en la que había un desarrollo económico continuo y una expansión de la protección social. Mañana volveré sobre las razones de lo que se empezó a llamar, a mediados de los años 70, “la crisis”, y que se convirtió en algo más que una crisis pasajera. En todo caso, a partir de este momento se puede hablar de un incremento de la inseguridad social. Es decir, de una dinámica inversa a la que se había impuesto sobre todo durante los años 30. 
No tengo tiempo de entrar en detalles sobre estas situaciones, que por demás son muy diversas, y que caracterizan a este incremento de la inseguridad social. Sin embargo, voy a subrayar que afectan tanto a las personas que trabajan como a las que no trabajan. A las personas que trabajan, porque hay un desempleo masivo que comienza a desarrollarse a partir de los años 70 y 80. También a las personas que están en búsqueda de un trabajo más o menos improbable, como por ejemplo, los jóvenes que tienen dificultades para rebuscárselas (galérer como se dice en francés); son personas que viven más o menos de trabajitos, de un poco de ayuda familiar o social, en algunos casos, de un poco de delincuencia también y creo que es una buena imagen de la inseguridad social contemporánea.

Esta inseguridad social comienza a referirse a las personas que trabajan y desde hace algunos años se ha empezado a hablar, en Francia, de trabajadores pobres. Antes se hablaba de los working poor puesto que se pensaba que era una situación reservada para los Estados Unidos y que en países como Francia no se veía este tipo de situación. Pero desde hace algunos años se observa efectivamente que hay personas que trabajan y que con su trabajo no alcanzan a tener suficientes recursos de base para garantizar su independencia y la independencia de su familia. Esto ocurre porque, a menudo, trabajan de manera precaria, alternando momentos de trabajo y de no trabajo, o bien con trabajos parciales, etc. Evidentemente esta inseguridad del trabajo tiene otras consecuencias, puesto que era con el trabajo, en particular en Europa, con lo que estaban ligadas las principales protecciones. Por lo tanto, la pérdida de la protección del trabajo genera una serie de consecuencias negativas sobre las que hablaré de manera más precisa mañana. Voy a tomar solamente el caso de la vivienda. Se puede observar un desarrollo de condiciones de mala vivienda, lo cual en una sociedad como la de Francia, que es de todas formas una sociedad bastante rica, representa algo negativo. Además, se han realizado cierto número de encuestas en la prensa y los periódicos sobre los trabajadores sin domicilio estable, que no podían, con su trabajo, pagar un arriendo para poder vivir de manera fija en algún sitio. Es un ejemplo extremo pero que ilustra bastante bien esta degradación que se ha producido en los últimos treinta años. La inseguridad social hace treinta años, la pobreza, aparecía como una fenómeno superviviente 
que se acabaría; luego, hacia los años 80, empezamos a hablar de los nuevos pobres. Es decir de personas que habían vivido una existencia más o menos normal, pero que no podían seguir y se encontraban en la pobreza. Hoy este fenómeno parece adoptar mayores dimensiones dado que hay incluso trabajadores pobres, lo cual creo que se puede interpretar como un efecto de este aumento de la inseguridad social que afecta a sectores cada vez más grandes de nuestra sociedad.

Mi intención no es hacer "miserabilismo”, ni dar una imagen catastrófica de la situación francesa, puesto que no olvido que en términos de pobreza, de precariedad del empleo, de ausencia de seguridad social, la situación es, sin duda alguna, mucho más grave aquí en Colombia. Pero como decía al principio, sentirse en inseguridad, vivir la inseguridad, no es algo que exista en absoluto. Lo que me parece característico de la situación francesa, y por demás europea, es esta conciencia de una degradación, dado que partimos de un alto nivel de protección y estamos experimentando esta especie de miedo, de retroceso, aun más cuando, les recuerdo, a estos miedos relacionados con la inseguridad social se agregan los que conciernen a la inseguridad civil que mencioné anteriormente. Creo que va a ser tiempo de detenerme por hoy puesto que quisiera que discutiéramos interrogantes sobre las dinámicas que originan esta inseguridad social. Quisiera, si ustedes así lo desean, disponer de un tiempo para abrir la discusión sobre lo que he dicho hoy y, en particular, sobre la diferencia entre inseguridad civil e inseguridad social, para saber si ustedes están de acuerdo sobre este punto. Creo que es una diferenciación muy sencilla, no se necesita ser genial para hacerla pero me parece importante reflexionar sobre lo que significa "estar protegido” en una sociedad moderna. Hay dos grandes tipos de protección: la que garantiza la paz civil y la que garantiza la seguridad social. Las dos son importantes, aunque también es importante no confundirlas, sobre todo si uno se plantea el interrogante de combatir la inseguridad, puesto que no es por los mismos medios que se puede luchar contra la inseguridad civil que contra la inseguridad social.

Se podría ilustrar de manera más concreta lo que digo puesto que en Francia tenemos “el problema de los suburbios” y tal vez este tipo de análisis pueda tener eco aquí. Se puede decir que si en Francia tenemos el problema de los suburbios, es porque estos territorios 
están asociados a dos tipos de inseguridad. Por un lado, altos índices de violencia, de delincuencia, de agresión contra las personas, índices más altos que el promedio nacional, pero también tenemos índices de desempleo, de trabajo precario más altos que en cualquier otra parte, y sobre todo un gran desempleo de los jóvenes. Así pues tenemos dos cosas que coexisten, lo cual hace que haya podido darse recientemente una cierta explosión. Pero si se trata de combatir esta inseguridad, podemos observar en Francia una tendencia que consiste en combatir exclusivamente la delincuencia, la violencia contra las personas, como si esta inseguridad civil fuera la totalidad del problema de la inseguridad. Me parece que aunque lográramos erradicar completamente la delincuencia, lo cual es por demás, sin duda, imposible, o partiendo de la hipótesis de que elimináramos la inseguridad civil, no se podría suprimir la inseguridad social provocada por el desempleo, la degradación de las condiciones de trabajo, etc. Me parece, entonces, que hay una especie de contradicción en las políticas de seguridad tal y como ellas están siendo aplicadas hoy en día en Francia. No sé si en Colombia también sucede lo mismo, haré la pregunta al respecto. Por un lado, el Estado debe ser fuerte, e incluso uno se hace una imagen del Estado como una especie de gendarme que debe ser despiadado contra la delincuencia y contra la violencia. Tal vez sea cierto, pero en todo caso en Francia se olvida el otro papel del Estado: el Estado social, el papel de protector del Estado. Por liberalismo se es completamente laxo, es decir que se deja desarrollar el desempleo, la precarización que mantiene la inseguridad social.

Este ejemplo muestra que una lucha contra la inseguridad sería una lucha en la que se asocien políticas de mantenimiento del orden con políticas sociales fuertes. Soy consciente de que es más fácil decirlo que hacerlo, pero pienso que haciendo esta diferenciación logramos tener una llave para apreciar las políticas que se aplican en nombre de la seguridad en Francia, aunque tal vez en Colombia también. En todo caso, se podría discutir esto. Discutir también la propuesta que he hecho, que consiste por un lado en desconfiar de una concepción globalizante de la inseguridad. Una actitud que ve riesgos por todo lado, y que considera nuestra sociedad únicamente como una sociedad de riesgo, adicionando todos los factores de la inseguridad: el miedo a ser agredido en la calle o a ser tomado como rehén, el miedo de perder el empleo, el miedo de contraer el cáncer al comer un producto. 
Pienso que es necesario decir, y lo someto a discusión, que la seguridad absoluta es un mito y que es incluso un mito peligroso, porque la vida comporta siempre lo imprevisible, y que en el fondo la seguridad absoluta sólo se encuentra en la muerte. Pienso que hay que desinflar la preocupación loca sobre la seguridad, pero al mismo tiempo, y no es contradictorio, hay que señalar la importancia fundamental de la necesidad de protección, porque al mismo tiempo el hombre tiene absoluta necesidad, me parece, de protección para vivir, tanto en la paz civil como con un mínimo de seguridad social. Por esto creo que la necesidad de protección, y en particular la necesidad de protección social, es fundamental. Es una reflexión que comienza hoy y que podemos continuar mañana cuando insista en el carácter profundamente desestabilizador de la falta de protección social sobre la organización del trabajo y sobre el estatus del individuo. Trataré de sugerir que una protección social fuerte es como el cimiento necesario de una sociedad democrática. Seguiremos entonces este tema mañana, pero podemos comenzar a discutir un poco ahora para saber si, en general, ustedes están de acuerdo o no, lo cual es perfectamente su derecho, con este enfoque.

\section{Preguntas y respuestas}

1. Una utopía contemporánea debe exigir una sociedad de pleno empleo. ¿El pleno empleo es posible en el capitalismo? Si los capitalistas tienen necesidad de un ejército de reserva, se podría creer que es imposible encontrar una solución al problema del empleo en el capitalismo.

Voy a tratar de contestar rápido. Creo que se puede constatar, y no es un juicio de valor, que el capitalismo en su forma actual no puede garantizar el pleno empleo, si se entiende por empleo una condición de trabajo sólida con salarios importantes o relativamente importantes y sobre todo con derechos sociales y protección social. Si digo esto, es porque es una constante en Francia. Desde hace veinte años existe el no empleo. Es decir, que existe una proporción de la población activa del orden del 10\% 
que, a pesar de sus esfuerzos por luchar contra el desempleo, se mantiene en el no empleo. Por lo tanto, creo que efectivamente hay no empleo. Pero además voy a insistir en que es a condición de dar a la palabra empleo su sentido fuerte, asociando las garantías del derecho al trabajo y a la protección social. Actualmente hay en Francia, y también en Europa, una especie de tentación de salir del no empleo institucionalizando formas que podríamos llamar de sub-empleo con relación a este estatus del empleo. Se trata de realizar actividades que se encuentran por debajo del empleo, en términos de la duración, el salario, los derechos sociales, la protección del trabajo. Sin duda, ustedes oyeron hablar, hace poco, del movimiento social que se produjo en Francia contra el “Contrato para el Primer Empleo” (CPE) y yo pienso que eso muestra bien lo que yo trato de decir aquí. La reacción al contrato para el primer empleo fue el rechazo por parte de un gran número de franceses que manifestaron en las calles contra este tipo de degradación del empleo, en el sentido de la precarización del empleo. De manera más general: no me parece, efectivamente, que el pleno empleo en el sentido fuerte del término se pueda dar en el régimen actual del capitalismo. Por en contrario, podríamos ir hacia una sociedad en la que las actividades se multiplicaran hasta tal punto que estuviéramos en una sociedad de plena actividad, sin que eso llegue a ser una sociedad de pleno empleo, en el sentido amplio de la palabra. Supongo que ustedes conocen mejor que yo el trabajo informal tan implantado en la sociedad colombiana. Pero en Francia es un retroceso a formas de ocupación inferiores a las que se habían desarrollado en las sociedades de tipo occidental. Es una diferencia importante sobre la que sería interesante reflexionar.

2. Se dice que Colombia aceptó una disminución del salario mínimo, con el fin de reducir el trabajo, ¿esto es un mito desmovilizador?

Es una pregunta sobre el hecho de que Colombia aceptó disminuir el salario mínimo. Primero que nada, no estoy aquí para juzgar la política de Colombia. Sencillamente y de 
manera más general, pienso que esta medida se inscribe perfectamente, bajo la inspiración del Banco Mundial, en una política mundial del todo económica, es decir, una política en la que el salario, en particular, debe ser la variable principal del ajuste para que las empresas puedan ser competitivas, rentables, etc. Es la política liberal que es sostenida por el Banco Mundial, entre otros. No hay que subestimar su fuerza, muy considerable. Simplemente, lo que se puede temer son los efectos sociales de este tipo de política. Me parece que detrás de eso hay una opción de sociedad. Y no se puede hacer una sociedad verdaderamente, en el sentido fuerte del término, simplemente en función de las leyes del mercado, de la competencia, de las ganancias por las ganancias. Eso introduce una competencia entre las personas que puede ser útil en cierto nivel, pero si está regulada mediante ciertas protecciones. Esta política liberal que se instaura en el nivel de la mundialización se apoya sobre reducciones de salarios y sobre reducciones de la protección de los derechos del trabajo que son considerados como obstáculos al libre desarrollo de las empresas. Personalmente, y no sólo por razones políticas, sino como sociólogo, pienso que esta opción unilateral por el todo económico es en extremo peligrosa, porque una sociedad no existe sin un mínimo de solidaridad, sin un mínimo de intercambios entre las personas que no sea sólo el imperativo de la ganancia. Una sociedad librada completamente a las leyes del mercado no es una sociedad en el sentido propio del término. Una sociedad librada a la competencia de todos contra todos sería más bien una jungla que una sociedad. Por lo tanto, creo que la exigencia de protección, de derechos, de reglamentación es fundamental para que haya sociedad. Eso debería conducirnos a relativizar esos imperativos del mercado, que por cierto son importantes y que se deben tomar en serio, pero en el marco de una especie de compromiso con un mínimo de seguridad y de protección para aquellos que también hacen funcionar el mercado también, es decir, para los trabajadores. 
3. ¿Cómo concibe usted la relación entre democracia y protección social? Si el desempleo y el sistema capitalista son la causa del problema social; ¿por qué combatir la consecuencia que es el problema social y no la causa de éste?

Pienso que es sensato porque el desempleo, a partir de cierto nivel, es peligroso para una democracia. Una democracia moderna no implica necesariamente una igualdad completa de condiciones, porque las sociedades modernas son sociedades muy diferenciadas y hay en ellas cierto grado de desigualdad. Eso se puede lamentar, pero es un riesgo de que eso sea un hecho. Además, no sé si hay que lamentarlo completamente, porque el ejemplo histórico que tuvimos de la imposición de una igualdad total de condiciones sociales, es decir la experiencia bolchevique, no fue una experiencia del todo feliz. Sea que se lo lamente o no, una democracia no es perfectamente igualitaria; pero debe ser una sociedad en la que cada uno tenga un mínimo de recursos y de derechos para hacer sociedad con los demás, es decir, para poder intercambiar en una relación de interdependencia y no solamente de dependencia. Tenemos ejemplos de esto. Voy a tomar unos ejemplos exagerados: si hay amos y esclavos, no es una democracia; si hay señores y siervos eso no es una democracia. A principios del siglo XIX, cuando había patronos muy poderosos y proletarios completamente miserables, no había una democracia en el sentido social de la palabra. Una sociedad en la que hubiera tasas de desempleo importantes, difícilmente sería una democracia, puesto que habría una masa importante de la población que estaría excluida de los intercambios sociales. Aunque no sea la igualdad de condiciones, una democracia es, al menos, una similitud de posiciones. El desempleo, al menos a partir de un cierto nivel, es peligroso para una democracia.

4. ¿Cuál es hoy el significado de los nuevos movimientos sociales ante la seguridad social, la seguridad civil y el Estado? Por otro lado, ¿cuál es la reacción del Estado ante los 
movimientos sociales actuales y cuál es la reacción de los movimientos sociales ante la reacción del Estado y los problemas de los suburbios?

Hay demasiadas preguntas como para que tratar de dar respuesta a todas. En pocas palabras diré, con relación a los movimientos sociales y a partir de este ejemplo reciente de Francia, que es muy significativa esta reacción al contrato de primer empleo (CPE) de la cual hablé antes. Es muy importante. Pienso que fue una respuesta relativamente inesperada pues antes de este CPE se habían tomado una serie de medidas que iban en el mismo sentido y que pasaron sin provocar grandes oleadas. En un momento dado hubo como una toma de conciencia por parte de muchas personas sobre el riesgo de la instalación en la degradación, de la precariedad de las relaciones de trabajo, y creo que este movimiento sorprendió a mucha gente. Fue una reacción fuerte ante dicha tendencia. Personalmente, reconozco que me alegro de esta reacción. Pero no hay que hacerse muchas ilusiones, porque las fuerzas que van en el sentido de esta precarización son extremadamente fuertes. De todas maneras, aunque no podemos profetizar sobre el futuro, pienso que el trabajo de un sociólogo es tratar de comprender lo que sucede, de hacer diagnósticos tan precisos como sea posible. No podemos saber lo que traerá el mañana y si este movimiento se desarrollará y tendrá consistencia para bloquear este movimiento de precarización del trabajo. Francamente no lo sé. Para establecer la relación con una de las preguntas que fue formulada sobre el Estado, diré que el problema de los movimientos sociales es importante; la sociedad civil y sus reacciones son importantes, pero pienso que no son realmente eficaces sino cuando se retoman en el nivel del Estado, puesto que el Estado tiene verdaderamente el poder de dar fuerza a la transformación social. Lo que constatamos con frecuencia es que los movimientos sociales se acaban bastante rápido. No quiero juzgar a los antiguos combatientes, viví Mayo del 68 que fue un gran movimiento social. Entre los efectos que tuvo, algunos fueron positivos, y otros lo fueron menos. En todo caso, hay un debilitamiento del movimiento social mientras no se traduce en decisiones que, a mi manera de ver, 
pertenecen al Estado. Sé que esta posición es discutible y tal vez yo sea jacobino como se dice en Francia, es decir demasiado apegado a cierto poder del Estado pero [...].

5. En su intervención en Argentina, usted dijo que la protección social es una responsabilidad del Estado y que las empresas no pueden intervenir en eso porque ellas se encuentran sometidas a una dinámica de competitividad en la que el asunto de la seguridad social no es la prioridad. En este contexto, ¿cuál sería el papel de las empresas con relación a la seguridad social?

Esto sería la prolongación de lo que traté de decir en la respuesta anterior. Es, por lo demás, el mismo tipo de pregunta. Yo no hablo mal de las empresas. Pienso que la empresa es un factor fundamental de la creación de la riqueza social y, al igual que decía antes, no se puede hacer como si el mercado no existiera, no podemos hacer como si las empresas no existieran. Nos queda por esperar que sean efectivamente eficaces, competitivas, etc. Sencillamente eso es una parte del asunto y no veo cómo podamos construir una sociedad a partir del modo de funcionamiento de una empresa. No podemos reprocharle a una empresa que sea competitiva, pero el asunto no es criticar a la empresa, sino de asegurar que ella no tenga todo el poder y que se establezca un tipo de compromiso social entre sus intereses y los de los trabajadores. Si estamos en una sociedad capitalista, hay dos posibilidades: o llegamos a vivir como en una jungla en donde todos están en contra de todos, o logramos construir esta especie de compromiso que no es fácil -es más fácil decirlo que hacerlo-, en el que los intereses de la empresa serían respetados pero también en donde habría en contrapartida -y por esto las protecciones son esenciales-, protecciones para los trabajadores. Podemos reconocer la presencia, la fuerza y la utilidad de la empresa, pero para que una empresa exista en una sociedad que sea una sociedad humana, no tiene que ser la ley de la sola empresa la que reine. 
Nos propusimos hacer una reflexión sobre la cuestión ¿qué es estar protegidos en una sociedad moderna? Ayer vimos que la protección se podía tomar en dos sentidos principales: la protección civil, que consiste en asegurar los bienes y las personas, garantizar la paz civil; y la protección social, que es garantizar un mínimo de recursos y de derechos necesarios para la independencia social de los individuos y permitirles satisfacer las condiciones de base de su existencia. También dijimos que, al menos en una sociedad como Francia, esta seguridad social había sido garantizada mediante el establecimiento de grandes sistemas de protección social, pero que se había producido, a partir de los años 70, una especie de inversión de la tendencia, y que se podía hablar a partir de ese momento de un aumento de la inseguridad social. Sobre eso mostré una serie de signos.

Ahora me gustaría extraer la lógica que está detrás de este aumento de la inseguridad social. Me parece que se puede interpretar como el cuestionamiento del compromiso social que se había construido en el marco del capitalismo industrial. El capitalismo industrial en su período de mayor crecimiento, después de la Segunda Guerra Mundial, había logrado llegar a cierto equilibrio entre los intereses del mercado, es decir la productividad y la competitividad de las empresas, y los intereses del mundo del trabajo, es decir, cierta seguridad y protección de los trabajadores. Como resultado de una larga serie de luchas y de conflictos, los trabajadores tenían cierta protección mediante su participación en formas de organización colectiva; para el trabajo, convenciones colectivas, regulaciones colectivas sobre el derecho al trabajo y a la protección social con la garantía del Estado, que es la instancia de lo colectivo por excelencia. Se puede decir, creo, que es lo colectivo que protege o que protegía a los trabajadores. Se produjo una especie de sinergia entre las formas colectivas de organización del trabajo del capitalismo industrial, con el peso de la gran industria y las 
exigencias de la producción en masa, lo cual dio lugar a sindicatos poderosos representantes de los intereses de las grandes categorías profesionales homogéneas, haciendo así, en cierta medida, contrapeso a las presiones del mercado. Esto permitió esta especie de equilibrio de conflictos entre, para decirlo en pocas palabras, los intereses del capital y los intereses del trabajo.

Esto fue sin lugar a dudas lo que salvó al capitalismo industrial, porque, como ustedes bien lo saben, la revolución no tuvo lugar en Europa Occidental y la relación de subordinación salarial no fue suprimida; un asalariado no trabaja para sí mismo sino para su empleador (un lenguaje marxista diría que se trata de cierta explotación del trabajador). Pero en contrapartida, el trabajador goza de protecciones sociales fuertes relacionadas con su trabajo, de derechos sociales como el derecho al trabajo y a las protecciones sociales, como por ejemplo, el derecho a la jubilación. Si quisiéramos hablar de política, podríamos decir que es una respuesta reformista que se impuso en Europa Occidental y, además, en el contexto bien particular que sobrevino después de la Segunda Guerra Mundial, en unas condiciones sobre las que sería bueno hablar un poco si dispusiéramos de más tiempo. En particular, sobre el hecho de que estos Estados de Europa Occidental —países como Francia, Gran Bretaña, Alemaniatenían una posición hegemónica sobre el conjunto del planeta y que los intercambios desiguales que ellos imponían a los demás países del mundo les permitieron desarrollar simultáneamente políticas económicas eficaces y políticas sociales ambiciosas. Es sin duda alguna la razón principal por la que estos sistemas de protección no se pudieron desarrollar en países de la periferia, como se les llama a veces, detenidos por esta desigualdad de intercambios en el mundo. Creo que podríamos decir que hubo una primera forma de globalización que se tradujo en una inmensa injusticia respecto a estos países periféricos y que sus secuelas todavía se sienten hoy en Colombia, por ejemplo. Porque este desequilibrio, primera forma de globalización, dio lugar a que aquí, y de manera más general en América del Sur, estos sistemas de protección no se pudieran desarrollar. 
En cambio, el sistema de protección funcionó bastante bien en los países europeos y, aunque se diga en términos políticos que es reformismo, el resultado condujo de todas formas a una transformación fundamental de la condición de los trabajadores. Para comprenderlo, bastaría con comparar, si tuviéramos tiempo, la situación del proletario a principios de la industrialización — que en Europa era terrible: miseria, inseguridad social, etc. - con la condición promedio de una asalariado europeo hacia 1960, por ejemplo, cuando contaba con un salario relativamente correcto, en general, y sobre todo, tenía seguridad hacia el futuro gracias a las garantías, precisamente, del derecho al trabajo y de la protección social.

Era ese equilibrio — por cierto no completamente ideal—, lo que era frágil y que fue puesto en crisis por el nuevo régimen del capitalismo que prevalece actualmente; un capitalismo más agresivo, que juega a la competencia exacerbada en una economía globalizada bajo la égida del capitalismo financiero internacional. Esto es el anuncio y el comienzo de otra forma de globalización de la cual los países occidentales no son ni serán los beneficiarios exclusivos, porque pierden una parte de su hegemonía en la medida en que este funcionamiento del nuevo régimen del capitalismo da al traste con las formas de organización y de protección colectivas que se habían desarrollado durante el capitalismo industrial. Se podría decir que la dinámica profunda de este nuevo capitalismo es una dinámica de descolectivización o de re-individualización. Me parece que es necesario comprender este punto, aunque pueda parecer un poco abstracto, para comprender las razones profundas del aumento de la inseguridad social y porque es algo que en Europa no se entendió de inmediato, sino hasta que se empezó a hablar de la crisis a mediados de los años 70. Al principio fuimos sensibles a las manifestaciones más evidentes, como el crecimiento de un desempleo masivo y el debilitamiento de las relaciones laborales, que son fenómenos importantes y graves. Pero me parece también que ahora, al ver las cosas con algo más de distancia puesto que ya hace un cuarto de siglo que empezó este proceso, comenzamos a constatar o a comprender que, sin duda, hay algo más profundo detrás del desempleo, detrás de la precarización de las relaciones laborales y que se trata del proceso de descolectivización 
y de re-individualización que mencioné. Un proceso que, por lo demás, se desarrolla en diferentes niveles. Ante todo, en el nivel de la organización del trabajo, en la que se asiste a una individualización de las tareas, que exigen movilidad, adaptabilidad, hacerse cargo de la responsabilidad por parte de los trabajadores. En lugar de la organización colectiva y jerarquizada, se exige una implicación personal del trabajador o la constitución de pequeñas unidades de trabajo que se supone que deben manejar su propia producción. En cuanto a las trayectorias profesionales, nos encontramos ante la misma movilidad. Ulrich Beck habla de un "modelo biográfico” para designar esta exigencia de que cada individuo se haga cargo de sí mismo en su trayectoria profesional: debe hacer elecciones, llevar a cabo reconversiones, hacer frente a los cambios. A diferencia de las carreras que se desarrollaban dentro de una misma empresa, las carreras se vuelven hoy discontinuas. También aquí es necesario que el individuo se movilice para asumir la gestión de su propia carrera profesional.

¿Cuáles son entonces las consecuencias de este proceso de individualización o de descolectivización? Creo que es necesario abstenerse de hacer una lectura únicamente catastrófica, porque es cierto que para muchas personas esta nueva forma de organización es beneficiosa. Logran liberarse de constricciones que podían ser muy pesadas, como en la organización taylorista del trabajo, maximizan sus oportunidades y expanden sus posibilidades. Se podría decir que resultan ganadoras en esta transformación actual de la organización del trabajo. Además, sobre estos buenos resultados se basa el discurso liberal que domina actualmente y que celebra el espíritu empresarial, la liberación de las constricciones, la expansión de las capacidades individuales, etc. Este discurso no es completamente falso; es válido para ciertas categorías de trabajadores, pero es unilateral. Este discurso silencia cosas; olvida que se ha de tener en cuenta la suerte de todos aquellos, muy numerosos por cierto, que no pueden adaptarse a esta nueva regla del juego, a esta movilidad. Y no es que sean necesariamente más estúpidos que otros, sino que no han tenido suficiente formación y no han sido suficientemente acompañados para poder enfrentar de manera positiva el cambio. Son personas que han sido superadas por los acontecimientos; la nueva 
coyuntura las ha invalidado. Por eso, pierden seguridad y, por lo general son, declaradas no empleables.

Voy a tratar de sacar algunas consecuencias principales de esta gran transformación, para utilizar la expresión de Karl Polanyi en el título de su célebre libro. Ayer decía que se podía traducir este cambio en términos de un aumento de la inseguridad social, pero voy a tratar de ser un poco más preciso analizando estos cambios un poco más de cerca en tres campos. El campo del estatus del trabajo o del empleo, el estatus del individuo, y el de la protección social propiamente dicha. Estas tres dimensiones se encuentran completamente ligadas, como procuraré hacer ver.

Comienzo por las transformaciones profundas que esta dinámica implica en el estatus del trabajo o del empleo. Con las transformaciones del trabajo que se operan hoy, asistimos a una especie de desmantelamiento del estatus del empleo. Pienso que el estatus del empleo es la forma dominante que había adoptado la organización del trabajo en Europa, es decir la estabilidad en la duración, lo que llamamos los contratos a duración indefinida, a los cuales se encuentran ligadas estas garantías del derecho al trabajo y de la protección social. Actualmente, además del desempleo, además de los períodos de alternancia entre empleo y desempleo, tenemos una multiplicación de contratos de duración definida, es decir contratos a término, trabajo subcontratado, trabajo parcial. Esto es lo que llamamos formas atípicas de empleo. Pero me pregunto si no hay que ir más lejos en el análisis porque, cuando se habla de formas atípicas de empleo, esto supone que el verdadero empleo es el empleo clásico con todas sus prerrogativas, las que acabo de enumerar. Creo que hay que preguntarse si lo que está sucediendo no es una redefinición o una recomposición bastante profunda de la noción misma de empleo. De hecho, además de estas formas de empleo, llamadas atípicas, vemos que también se desarrolla una serie de actividades de las que podemos decir que están más allá del empleo; un conjunto de empleos degradados, empleos especiales para los jóvenes. No voy a entrar a enumerarlos, pero en esta serie de nuevas formas de actividad, la última fue el intento del gobierno francés de instaurar el "contrato de primer empleo” (CPE) que, con la intención de disminuir el número de jóvenes 
desocupados, creaba una forma de vinculación laboral de menores de 26 años que restringía severamente los derechos laborales. Sin duda, ustedes oyeron hablar, hace poco, del movimiento social que se produjo en Francia contra el CPE y que impidió que se instaurara. Pienso que eso muestra bien lo que yo trato de decir aquí: la reacción al contrato para el primer empleo fue el rechazo por parte de un gran número de franceses, que manifestaron en las calles, contra este tipo de degradación del empleo, de precarización del empleo. Se trataba de personas que han comenzado a comprender que esta derivación de la noción de empleo planteaba problemas considerables y aparecía como inaceptable. El propósito del CPE no era una innovación. Estamos en trance de deslizarnos desde una definición del empleo como algo sólido, estable, hacia unas actividades que ofrecen la ventaja de dar acceso al trabajo, pero con unas contrapartidas muy por fuera de las ofrecidas en el empleo clásico, en cuanto a la duración, la remuneración, el salario y los derechos sociales. De tal forma que sería necesario redefinir, o al menos reflexionar de nuevo, sobre lo que se entiende por precariedad. En un primer momento, cuando empezamos a hablar de precariedad, pensamos en unas carencias con relación al empleo que eran más o menos provisionales, y luego en períodos de precariedad bastante largos, pensando que se volvería al empleo normal. Pero no parece que sea lo que está sucediendo actualmente; vemos que se desarrollan múltiples formas de actividades que están por fuera, por debajo, de lo que, hasta hace poco, llamábamos empleo. De tal forma, por lo demás, que se podría concebir que, más allá del desempleo, llegaríamos a una sociedad de plena actividad, es decir, que todo el mundo haría algo pero sin que se tratara de verdaderos empleos. Se ejercen presiones muy fuertes en esta dirección, que consisten en hacer trabajar bajo cualquier condición, a cualquier precio y en una especie de precariedad permanente. Supongo que lo que describo de manera rápida aquí, y que parece instaurarse actualmente en un país como Francia o en Europa, se asemeja mucho a lo que ustedes llaman aquí el trabajo informal y que, al parecer, conocen desde hace mucho tiempo.

Puedo sacar otra consecuencia de estas transformaciones que están relacionadas con las transformaciones del trabajo, pero que se refiere a la degradación del estatus del 
individuo. Es un leitmotiv de la reflexión sociológica de hoy decir que nos encontramos, cada vez más, en una sociedad de individuos, que el individuo es el valor de referencia de nuestras sociedades, y que, en general, exaltamos al individuo, su sentido de responsabilidad, su espíritu empresarial y que , como decía hace un momento, el corazón de la ideología actualmente dominante es la idea de liberar al individuo de las obligaciones burocráticas, del Estado, etc., para permitir que desarrolle todo su potencial. Pero me parece que ésta es una concepción ingenua de la noción de individuo, como si se tratara de una especie de substancia que tuviera por sí misma la capacidad de realizarse, de existir. Me parece que la historia social muestra que no hay un individuo en sí, que un individuo, si puedo utilizar esta expresión, no se mantiene de pie por sí solo. Es necesaria la existencia de soportes o de puntos de apoyo para poder llegar a ser de manera positiva un individuo. Así, a comienzos de la modernidad, el individuo tiene necesidad de ser propietario para existir, para ser reconocido como ciudadano, y aquél que esté por fuera de la propiedad, como el proletario, que no tiene más que la fuerza de sus brazos para sobrevivir, aquél que no tiene nada, literalmente hablando, no es nada socialmente, no tiene ningún derecho, ninguna consideración. Se encuentra al margen de la sociedad, estigmatizado y subestimado. Este tipo de individuo es considerado individuo por abuso de lenguaje, como se habla del individuo de la Declaración de los Derechos del Hombre y del Ciudadano, por ejemplo. Pero estos individuos llegaron a convertirse en individuos completos cuando adquirieron protecciones y derechos. Es lo que yo había propuesto ${ }^{1}$ llamar una propiedad social, es decir, nuevos apoyos que se constituyen como una propiedad para la seguridad, como el derecho a la jubilación, que evoqué ayer. El derecho a la jubilación ofrece los recursos básicos para mantenerse independiente socialmente cuando uno no puede seguir trabajando. Permite que el individuo no sea excluido de los intercambios sociales y que no caiga en la degradación social. Podríamos decir que estos derechos sociales, como el derecho a la jubilación, es en resumen la propiedad de los no-propietarios y es gracias a

\footnotetext{
${ }^{1}$ Ver Robert Castel-Claudine Haroche, Propiedad privada, propiedad social, propiedad de sí mismo, Homo
} 
estos derechos sociales como los no propietarios han sido rehabilitados socialmente y pueden ser reconocidos como miembros completos de la sociedad. Es lo que podemos llamar una ciudadanía social, es decir, ser reconocido en paridad con sus conciudadanos, compartiendo los mismos derechos.

Comprendemos bien que la pérdida de estos bienes sociales, de esta propiedad social, destruye lo que se encuentra en la base de la existencia positiva del individuo, como sucede con el desempleado de largo tiempo o con los jóvenes que fracasan constantemente en la búsqueda de un empleo. Ellos no cuentan con estos apoyos que constituyen la independencia social del individuo y se convierten en lo que podríamos llamar individuos por defecto. Son individuos porque tienen sentimientos, afectos, necesidades, deseos, como todo el mundo, pero les faltan los recursos básicos para desarrollar de manera positiva la capacidad de ser individuos. Creo que podríamos decir que se ha producido, como uno de los efectos de esta crisis que evoco, una especie de bifurcación en la trayectoria del individuo moderno. Es cierto que estamos en una sociedad de individuos, que las personas son cada vez más individualistas, pero eso puede tener consecuencias positivas para algunos y negativas para otros. A través del proceso de individualización, hay individuos que pierden la capacidad de ser responsables, independientes, dueños de su destino. Les falta esta especie de propiedad social que les ofrecían los derechos sociales.

Ahora paso al tercer efecto de estas transformaciones que me parece importante, y que se produjo después del cambio de régimen capitalista. Es una modificación de la estructura misma de la protección social. Actualmente, mediante lo que llamamos protección social, se asiste a un deslizamiento de las protecciones de alcance general, que conciernen al conjunto de la población y que se dirigen a ese conjunto, hacia protecciones delimitadas y apuntan a categorías particulares de la población porque carecen de recursos y, lo que es por otra parte lo más frecuente, porque se encuentran por fuera del orden del trabajo o porque no logran incluirse en el trabajo. Es la lógica de 
lo que se llama los "mínimos sociales”. Es decir, que se da un mínimo de ayuda a poblaciones que se caracterizan por un déficit. Es una concepción que se podría calificar de minimalismo de la protección social. Porque en todo caso, en sociedades como las europeas, que son de todas formas sociedades ricas o relativamente ricas, no se tolera que las personas estén desprovistas de todo; entonces, se les acuerda un mínimo de recursos y de esta forma se evita que estén en una necesidad absoluta. Así, tenemos un cierto número de recursos como el Ingreso Mínimo de Inserción, el RMI, por su sigla en francés. Son retribuciones, subsidios a los que se tiene derecho, aunque no trabaje y a condición de no tener ningún otro recurso. Pienso que es una redefinición de la protección social. No es condenable en sí, yo no estoy aquí para condenar lo que sea, pero podemos decir que este tipo de medida es mucho mejor que nada y creo que, además, habría que comparar este tipo de medidas con las que se implementan en América del Sur, en Colombia, por ejemplo, en el marco de los programas del Banco Mundial, con relación a la pobreza. Creo que tenemos que discutir sobre esto. No podemos, una vez más, condenar este tipo de medidas, porque cuando las personas tienen realmente necesidad, la protección social como nivel mínimo de recursos es mejor que nada. En todo caso, creo que es necesario constatar que es una regresión importante respecto a la concepción ambiciosa o maximalista de la protección social que presenté al hablar de los países de Europea Occidental. Esta regresión es peligrosa para la cohesión social, puesto que introduce una especie de divergencia entre los ganadores y los perdedores de esta transformación en curso. Hay ricos que se vuelven más ricos, y ustedes conocen este proceso, y pobres que se vuelven más pobres, para expresarlo de manera esquemática.

Creo que lo que está en juego detrás de estas transformaciones es la posibilidad de constituir "una sociedad de semejantes". Es una expresión que había tomado de un autor francés, eso importa poco, se llamaba Léon Bourgeois, que definía finalmente la democracia como una sociedad de semejantes. Porque una democracia no es necesariamente una sociedad en la que existe una igualdad total de condiciones, porque hay diferenciación social, hay diferencias y tal vez haya cierto nivel de desigualdad, si 
las desigualdades no son muy grandes y pueden ser justificables. Lo que es escandaloso y que podría ser mortal para la capacidad de continuar constituyendo una sociedad es que el conjunto de los ciudadanos no tenga un mínimo de recursos ni de derechos para mantenerse ligado a los demás en relaciones de interdependencia y se encuentren completamente excluidos del orden de los intercambios sociales. Creo que la protección social en el sentido fuerte que he tratado de caracterizar es esta especie de cimentación entre los miembros de una sociedad que hace que ella constituya una sociedad de semejantes ligados por relaciones de interdependencia entre ellos y no solamente por relaciones de dependencia. De manera que, para que podamos reflexionar sobre la protección social, en el fondo, hay dos sentidos bastante diferentes que se pueden dar a la protección social. Está la protección social como ayuda particular que se da a los individuos menos favorecidos y más desprovistos, y que está en el nivel de la asistencia; y está por otro lado la protección social que se da a todos o que debería darse a todos, porque es para los que carecen de propiedad privada la condición de constituir la base para su ciudadanía social. Este es el punto que someto a discusión: por un lado, la noción de protección social extensa y fuerte para todos los miembros de la sociedad me parece esencial, pero también el hecho de que, con relación a la situación de hace unos treinta años, cuando se habían impuesto en gran escala estas protecciones en Francia y en Europa Occidental, nos encontramos hoy en una situación nueva. Dado el nuevo régimen del capitalismo al que me refiero, Francia y el conjunto del planeta se encuentran atrapados en esta nueva lógica de la búsqueda del beneficio por el beneficio; una lógica según la cual las protecciones asociadas al trabajo aparecen como muy costosas, y las regulaciones de la protección social y del derecho al trabajo aparecen como obstáculos para el libre desarrollo de las empresas y para la búsqueda de una competitividad absoluta. Me parece que esta lógica actúa en Francia y en Europa, como también en Colombia, en África y en América del Sur, porque estos países no están en planetas distintos. Podríamos plantear una hipótesis, y es que las diferencias entre estas situaciones en los países, que creo, ustedes llaman del primer mundo y los de la periferia, están en tren de esfumarse, porque los privilegios de los países del primer 
mundo están siendo cuestionados por la mundialización y estas diferencias tienden a atenuarse, de tal forma que podría haber una especie de homogeneización por lo bajo, debido al proceso de mundialización. Sin embargo, estoy llevando esta hipótesis al extremo, porque es evidente que las diferencias entre, por ejemplo, Colombia y Francia siguen siendo enormes y me parece que la razón principal o una de las razones principales de esta diferencia es la siguiente: Francia al igual que los países de Europa Occidental, había tenido tiempo de construir estos sistemas de protección cuya importancia he resaltado y, por lo tanto, fue un largo proceso durante alrededor de un siglo que ha pasado por bastantes luchas y conflictos, a través de los cuales se constituyó en Europa lo que se podría llamar una “cultura social” que todavía tiene cierta fuerza. Esto se puede ver con el movimiento social que logró el rechazo del “contrato de primer empleo” (CPE), porque este contrato era típicamente una medida que se inscribía en esta política de reducción de las protecciones ligadas al trabajo y al derecho del trabajo. A este respecto, se ha asistido recientemente a un retroceso importante de las fuerzas del liberalismo, que permite pensar que tal vez no todo se ha jugado en el sentido de la totalidad de lo económico, precisamente por la fuerza todavía muy grande de esta cultura de lo social que acabo de evocar y que hunde sus raíces en esa larga historia de luchas sociales. Me parece que en Colombia la situación es muy diferente porque esta cultura de lo social no ha tenido tiempo de conformarse. No es porque no haya habido luchas sociales, pues pienso que han sido tan fuertes aquí como en Europa. Pero las condiciones del compromiso social del capitalismo industrial no se han reunido en Colombia ni en América Latina, de manera general, por razones que habría que discutir; pero sin duda porque la clase obrera colombiana y las clases medias asalariadas no han tenido el poder y no han tenido el tiempo de conformar esta fuerza de equilibrio que obliga a las clases dominantes a establecer compromisos que permiten desarrollar sistemas amplios de protección social. Pero, es posible que me adelante demasiado, teniendo en cuenta mis conocimientos limitados sobre Colombia. Presento esto como una hipótesis y habría que discutir al respecto, porque si me he visto obligado a hablar sobre todo de Francia, es porque no podía hacerlo de otra forma por 
falta de conocimientos suficientes. Espero que estas dinámicas que se han dado en Francia les permitan reflexionar también sobre lo que sucede aquí, aunque sea estableciendo la diferencia e incluso la oposición. En todo caso, les agradezco, una vez más, la atención y la paciencia.

\section{Preguntas y respuestas}

1. ¿Qué es para usted la flexibilidad de los contratos de trabajo? ¿Esta flexibilidad de los contratos es un fenómeno positivo o negativo para el mercado de trabajo?

Con relación a esta pregunta sobre la flexibilidad de los contratos de trabajo, me parece que la aceptación de una cierta flexibilidad es necesaria porque si el análisis que ya presenté sobre la salida del capitalismo industrial no es falso, quiere decir que hay movimientos irreversibles que se han producido en la economía y que van en este sentido de la movilidad, de la individualización, de situaciones a las que no podemos darles la espalda. No podemos mantener formas de regulación que fueron convenientes para el capitalismo industrial. Es necesario suavizar las condiciones de acceso al trabajo, y también las posibilidades de cambiar de trabajo, etc. Pero el problema principal me parece que es que no siempre deben ser los mismos los que paguen, por así decirlo. Es decir que en nombre de esta flexibilidad, de la utilidad de la flexibilidad, no se acompaña a las personas a adaptarse a estos cambios, que para ellas la flexibilidad se traduce en una pérdida de estatus, una pérdida de derechos e, incluso, una eliminación mediante el hecho de que son declaradas como personas que no pueden ser contratadas. Entonces, hay que aceptar la movilidad, pero esta movilidad, esta flexibilidad debe estar asociada a nuevos derechos, de tal forma que el trabajador pueda, de manera positiva, asumir los cambios. ¿Qué quiere decir esto? Que el trabajador esté acompañado, por ejemplo, cuando se ve obligado por razones económicas a cambiar de empleo o a reciclarse a nuevas formas de empleo. Que haya durante estos períodos un derecho a 
recibir una remuneración, de acceder a una verdadera formación completa para asumir dicha flexibilidad. Es lo que los daneses han tratado de implementar y a lo que ellos llaman "flexi-seguridad". No es fácil de implementar, supone un gran número de problemas en su aplicación, pero me parece que esta es la dirección que hay que tomar. Sería un suicidio, sin duda, rechazar, de plano, las necesidades del cambio en el sentido de una flexibilidad en la organización del trabajo, pero es necesario exigir que esta trayectoria más móvil tenga una seguridad.

2. ¿Cómo hoy día en Francia se establece la relación entre el estatus del individuo y el estatus del trabajo? ¿Cómo está ligado el estatus del individuo al del trabajo?

Creo que se pueden observar estrechas correspondencias entre el estatus del trabajo y el estatus del individuo. Es decir, que si el estatus del trabajo es consistente, eso da una base para una consistencia de la personalidad social del individuo. Sobre todo, vuelvo a repetir, pienso que el trabajo es algo más que el trabajo; para mí el trabajo no es solamente una actividad, sino los derechos y las protecciones que están ligadas al trabajo, y como traté de mostrarles, estos derechos y estas protecciones ligadas al trabajo dan una consistencia a la mayoría de los individuos en las sociedades de nuestro tipo. Inversamente si el trabajo, la consistencia del trabajo se desmorona, el estatus del individuo se podría desmoronar en la medida en que reposa sobre estas protecciones y estas garantías que obtiene de su trabajo. Pienso, entonces, que hay una correlación bastante profunda entre los dos, ya sea en un sentido positivo o negativo.

3. Si bien la protección debe ser ofrecida por el Estado, ¿qué papel jugarían los sectores llamados terceros, las ONG, para garantizar la oferta de esta protección social? 
Si entendí bien la pregunta, está relacionada con el papel de las ONG y se trata de saber en qué medida las podemos interpretar como protección social. Creo que se puede hacer en cierta medida, pero a condición de diferenciar los sentidos de la protección social. Lo repito, he insistido mucho sobre esta idea de que el sentido fuerte de la protección social me parece ser el que pasa por los derechos sociales bajo la garantía del Estado y por lo tanto no están relacionados con el tipo de trabajo que promueven las ONG. Sin embargo, éstas pueden proteger a los individuos en situaciones particulares, pero más sobre la forma de ayuda, la filantropía, lo facultativo, cosas que pueden ser importantes e incluso esenciales, sobre todo, en situaciones un poco trágicas o dramáticas, en las que su presencia es absolutamente indispensable y debe ser reconocida. Pero este no es el sentido maximalista de la protección social que he tratado de caracterizar. Lo cual no implica de ninguna manera una crítica a estas intervenciones, pero se podría decir que se hacen en un registro privado, o casi privado, y que no tienen la consistencia y la cobertura de las protecciones sociales que pasan por la garantía del Estado.

4. Si usted habla de la necesidad de que el Estado garantice un mínimo de derechos sociales, ¿eso implica que es necesario pasar de un sistema capitalista a un sistema socialista, o hacia el socialismo? ¿El hecho de garantizar un mínimo vital no iría contra la obligación del ciudadano de buscar un empleo? ¿Cuáles son las consecuencias a nivel de la protección social que se derivan de los múltiples cambios en el sistema educativo francés?

Me disculpan pero no puedo responder a todas las preguntas o en todo caso, a algunas de las preguntas que exigen varias horas en su respuesta y de las que no disponemos. Para presentar, por ejemplo, el papel del sistema educativo con relación a las preguntas sobre las que discutimos hoy, necesitaría varias horas, por lo tanto me disculpan pero no voy a responder a esta pregunta. En cambio, voy a tratar de contestar 
a la primera pregunta planteada porque en este sentido es necesario evitar el contrasentido. La posición que yo defiendo no es el socialismo, aunque simpatizo más, como ustedes habrán podido notar, con la izquierda. Pero no es en esta dimensión política del Estado social que yo tomo las cosas. Por ejemplo, este Estado social se desarrolló fuertemente en Francia, bajo el régimen de De Gaulle. El Estado Gaullista presidido por el General De Gaulle, quien no era un hombre de izquierda, pero había entendido que para garantizar la cohesión en Francia, su modernización, era necesario acompañar las medidas económicas que habían sido adoptadas en el sentido de esta modernización con medidas sociales bastante fuertes. No es socialismo en el sentido partidario de la palabra, un Estado Social es efectivamente un Estado que toma la responsabilidad de garantizar una especie de ciudadanía social mediante unos derechos, no es necesariamente socialismo, en todo caso en el sentido en que el socialismo pasaría por la destrucción del capitalismo. Quiero agregar un punto más, desafortunadamente, no tenemos mucho tiempo, pero creo que no hay que confundir lo que yo llamé hace un rato la propiedad social, que está relacionada con los derechos sociales, con la propiedad colectiva. Podríamos en cambio mostrar que si en Europa ha prevalecido la respuesta reformista, de la que hablé antes, es esto lo que en cierta medida salvó al capitalismo, es decir que economizó, si se puede decir, la revolución. Es decir, economizó la colectivización de la propiedad, la supresión de la propiedad privada. ¿Por qué? Es cierto que la propiedad privada no fue suprimida, la propiedad colectiva no se instauró, pero se instauró lo que yo denomino la propiedad social, es decir, esos derechos y esas protecciones que se dieron a todos y que permiten suplir la ausencia de la propiedad para la protección de los individuos, pero que también, se podría decir, economiza la supresión de la propiedad privada, la colectivización, es decir, la opción radical del lado del socialismo, el comunismo. Entonces, pienso que políticamente esta posición no es un socialismo extremo, sino más bien el sentido de responsabilidad del Estado que garantiza la cohesión social, que puede ejercer un gobierno de inspiración socialista ciertamente, pero que también puede ejercer un gobierno que no sea de tendencia de izquierda, y para ello tomé el ejemplo del Gaullismo. En esa época, no 
tenía mucha simpatía por De Gaulle, incluso me oponía al Gaullismo. Sin embargo, reconozco, tal vez un poco tarde, que este Estado Gaullista hizo mucho por la protección social y al final el resultado fue bastante importante y positivo, poniendo entre paréntesis la coloración política, politiquera de este gobierno.

5. ¿Qué explicación daría a la fuerte movilización de esta juventud francesa durante los últimos meses frente a este sistema de protección social? ¿Considera usted que la juventud francesa tiene un movimiento social? ¿Estos movimientos sociales pueden domesticar la globalización y el mercado?

Yo no soy tan ingenuo para creer que los movimientos sociales puedan domesticar profundamente el mercado. Tomé como ejemplo las reacciones al Contrato del Primer Empleo para decir que estas reacciones no eran tan esperadas y que fueron muy fuertes y por la primera vez, sin duda alguna, desde hace mucho tiempo, permitieron interrumpir este movimiento de degradación de los contratos de trabajo y las condiciones de trabajo. Entonces, considero que es positivo. No considero, por ello, que haya que hacerse muchas ilusiones al punto de considerar que se trata de algo que pudiera cambiar una tendencia que se ha inscrito dentro de unas dinámicas profundas. Pero diría que es positivo y que falta ver cuáles serán las consecuencias a largo plazo. Preciso sencillamente que no fue un movimiento exclusivo de la juventud francesa y que si tuvo un éxito relativo fue porque se llevó a cabo mediante una alianza, un funcionamiento común entre los jóvenes, es decir, los estudiantes de colegio, de universidad y las organizaciones sindicales. Este es un punto con una importancia relativa en estos hechos, puesto que por primera vez en Francia, desde hacía mucho tiempo, hubo una unión sindical real, cuando uno de los dramas o de las debilidades en Francia, desde el punto de vista social, es que los sindicatos en general son débiles. Hay más o menos un 10\% de sindicados en la población activa, y además están divididos, y se hacen más o menos competencia entre ellos. En esta ocasión, hubo unión sindical 
entre los distintos sindicatos de asalariados y también una alianza con los jóvenes estudiantes de colegios y universidades. Es sin duda alguna esta conjugación lo que permitió a este movimiento tener cierto éxito. ¿Cuál es el alcance a largo plazo de este éxito? Eso es otra cosa y no creo que podamos predecirlo hoy.

6. ¿Apoya usted la idea de un salario mínimo sin relación con el lugar de trabajo, es decir, un salario independiente del lugar de trabajo defendido por cierto socialistas? ¿Cuáles serían los efectos, positivos o negativos, de este tipo de salario?

Esta idea no es defendida solamente por ciertos socialistas, también es defendida por personas de derecha, personas de izquierda, en una especie de confusión bastante grande. Desafortunadamente, contamos con poco tiempo para discutir a fondo este problema difícil e importante. Voy a dar de manera un poco brutal mi opinión. Pienso que las protecciones más fuertes son las que están ligadas al trabajo y toda la historia social lo demuestra. Las personas tienen protecciones fuertes ligadas al trabajo y por lo tanto es demasiado peligroso desconectar el trabajo de la protección. Pero podría ser que si las condiciones de trabajo se degradan tanto que no se le puedan relacionar protecciones suficientes, sería necesario, tal vez, pensar en otra posibilidad, y creo que es la posición de las personas que defienden este tipo de ingreso. Pero me parece demasiado peligroso, porque de las cosas una: o bien este tipo de ingreso, que no estará ligado al trabajo, sería de una especie de consistencia, no voy a dar cifras, pero sería un ingreso suficiente para .permitirle a las personas vivir de manera relativamente digna y decente, y en este caso, podría ser defendido. Esto querría decir que cada uno tendría recursos básicos suficientes, independientemente del trabajo. Eso me parece defendible en sí, pero me parece políticamente imposible. No se puede pedir, en Colombia ni en cualquier parte del mundo, dar un subsidio consecuente con las personas fuera del trabajo, porque los recursos son limitados y ningún partido político, cualquiera que sea, podría imponer eso, se suicidaría inmediatamente. Si hubiera un ingreso de este tipo, 
sería verdaderamente mínimo. Sería un ingreso de supervivencia, que, tal vez dirán algunos, es mejor que nada, y sin duda será mejor que nada, pero no será una verdadera protección, o en todo caso, será una protección muy inferior a las protecciones relacionadas con el trabajo. Al menos en Europa actualmente, no pienso que se haya perdido completamente el partido de tratar de defender las protecciones relacionadas con el trabajo. Soy pues muy crítico con relación a esta posición, reconociendo que no es una posición tonta y que tal vez se intentaría imponer si se supiera que definitivamente no se puede defender nada más serio en el ámbito de las protecciones relacionadas con el trabajo. 